\title{
Design of Lyapunov Based Nonlinear Position Control of Electrohydraulic Servo Systems
}

\author{
Edvard Detiček* - Mitja Kastrevc \\ University of Maribor, Faculty of Mechanical Engineering, Slovenia
}

This paper studies the nonlinear closed loop control of an electrohydraulic servo system. The control strategy is developed based on Lyapunov theory of nonlinear systems using integrator backstepping approach. Highly nonlinear nature of electrohydraulic servo system is well known. Main reasons for nonlinear and non-differentiable mathematical description of systems dynamics are the fluid compressibility, leakage flows, friction forces and nonlinear fluid flow through servo valve orifices. These nonlinear terms influence also the dynamic errors of the control system.

Two different nonlinear design procedures are employed feedback linearization and integrator backstepping. Backstepping is used here because it is a powerful and robust nonlinear strategy. These techniques are used for construction of nonlinear control algorithm. The effectiveness of it, to stabilize any operating point of the system is proved by computer simulation. The systems error dynamics significantly depends on tuning parameters of the controller. The wrong selection of these parameters may lead to saturation or chattering of control signals. All derived results are validated by computer simulation of a nonlinear mathematical model of the system. The results are also compared to these obtained with a conventional $P$ controller to prove that classic linear controllers fail to achieve a good tracking of the desired output, especially, when the hydraulic actuator operates at the maximum load.

The research studies represented in the paper shows big potential of Lyapunov based nonlinear controller design procedures, to obtain desired control objectives.

Keywords: nonlinear control, Lyapunov methods, integrator backstepping, position control, electrohydraulic servo system, computer simulation

Highlights

- The nonlinear mathematical model of electrohydraulic position servo system was considered.

- Simplifications of mathematical model according to specifications of real components.

- Lyapunov based position control is developed.

- $\quad$ The results of computer simulation compare the dynamic behavior obtained by conventional PID $(P)$ controller and Lyapunov based nonlinear controller.

\section{INTRODUCTION}

Electrohydraulic servo systems take important place in modern industrial automation. It has been used in many kinds of mechanizations, including robots, computer numerical control (CNC) press brakes, computer controlled testing machines, etc.

Electro hydraulic actuator system has become one of the most important actuators in the recent decades. It offers many advantages such as good capability in positioning, fast and smooth response characteristics and high power density. Due to its capability in positioning it has given a significant impact in modern equipment for position control applications [1] and [2]. The applications in position control can be found in production assembly lines, robotics, aircrafts and testing equipment [3] and [4]. However, excellent positioning in these applications requires an accurate electro hydraulic actuator. Therefore, the development of suitable controller which could reflect such characteristics is very significant, although the dynamics of electro hydraulic servo system is highly nonlinear [5] and [6].

In ordinary feedback systems the method of feedback linearization is used to eliminate nonlinearities. Feedback linearization employs changes of coordinates to transform a given nonlinear system into equivalent linear one. A major advantage of feedback linearization approach is related to the cancelations of systems nonlinear dynamics that are introduced in design process. On the other hand some kinds of nonlinearities can have positive effects on system stability, therefore their cancellation can lead to instability in the presence of modelling uncertainties. As a solution to this problem the integrator backstepping approached is proposed. The fundamental concept of backstepping method is introduced by Krstic et.al. in their book [7].

The approach focusing on the stabilization problem in stochastic nonlinear systems is developed in the extension of this book. The backstepping control method is also presented in [8] to [10] where 
this technique is explained in detail for regulating and tracking problem.

There are numerous of applications in industry, where the backstepping approach was used to obtained successful control of electric machines, wind turbines, based power production, robotic production systems and flight trajectory control.

Integrator backstepping control method allows also finding a solution for optimal control problems [11], estimating parameters and adaptive control design, as well as, development of robust nonlinear controllers. The observer based backtepping technique has been designed for force control of electrohydraulic actuator system [12]. The developed control system combine backstepping observer with adaptive and sliding mode controller. Electrohydraulic active suspension control system is also designed by backstepping approach [13]. To obtained best value for its tuning parameters the paricle swarm optimization tool is proposed in [14].

It is proved that the technique improve the transient stability and damping presented in the system. The performance of backstepping controller also depends on its gains or controller parameters. In this paper, the controller is developed on the basis of simplified mathematical model, while the parameters are tuned manually to prevent the control signal chattering.

\section{ANALYSIS AND MATHEMATICAL MODELLING OF THE ELECTROHYDRAULIC SERVO SYSTEM}

A typical electrohydraulic positioning servo system consists of a hydraulic power supply e.g. constant pressure hydraulic pump with relief valve and accumulator, a flow control servo valve (SV), a hydraulic cylinder, a position sensor and an electronic controller unit (Fig. 1).

Before of closed loop controller design for electrohydraulic servo system, the analysis of the system dynamics is required, as well as, mathematical modelling and computer simulation of dynamic behaviour [15] and [16]. The complete mathematical model of the system is based on physical laws that expresses dynamic behaviour and are described through differential equations. However, this task is troublesome due to the multidisciplinary nature of the electrohydraulic system that requires electric, magnetic, mechanical and hydraulic knowledge. Meanwhile, the nonlinearities of this type of system, such as fluid nonlinear properties, nonlinear servovalve dynamics and flow rate characteristics, as well as, nonlinearities associated with hydraulic actuator by converting hydraulic power into mechanical power, increase a model complexity. The methodology in this work is that a theoretical model is presented first, where each individual component is expressed in a phenomenological description context e.-g. basic physical law.

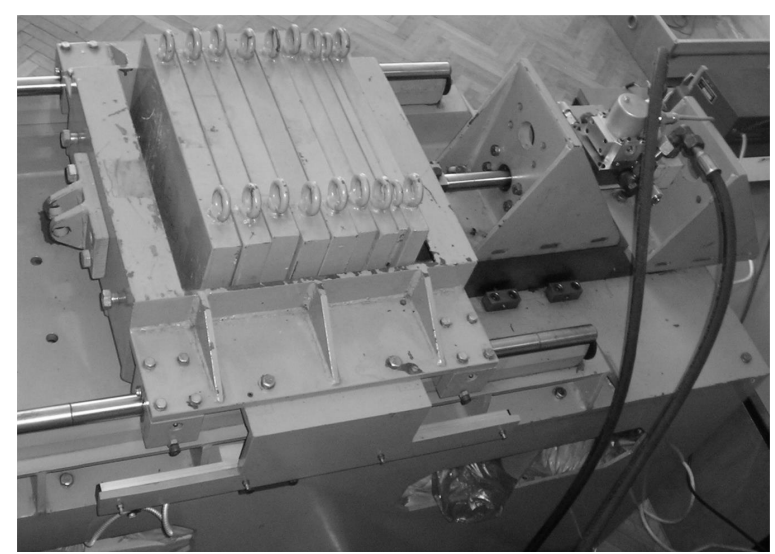

Fig. 1. A one degree of freedom electro-hydraulic servo system with load

\subsection{Description of the SV Dynamics}

Two stages SV consist of three main parts: the electrical torque motor, the hydraulic amplifier and the valve spool assembly (Fig. 2). In these SVs, the motor torque is commanded by an electrical current resulting in the flapper plate movement from its central position. The first stage is the so called flapper-nozzle system which allows the spool motion by adjusting the flapper via a low power electrical signal. The second stage relates the small spool motions $([\mu \mathrm{m}])$ into a large fluid flow through the spool ports.

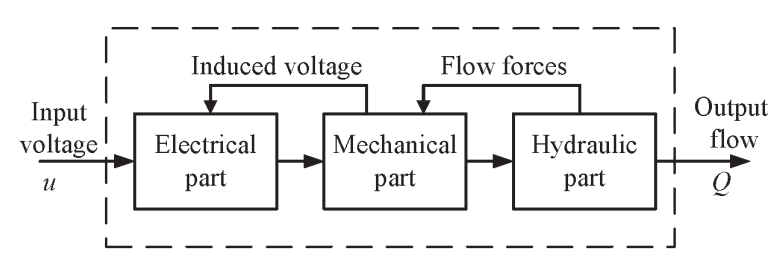

Fig. 2. Two stage SV consist of three main parts: the electrical torque motor, the hydraulic amplifier and the valve spool assembly

The flows in the SV concerns two parts: one is involved in the pilot stage and the other in the second stage.

In the first stage, the flapper deflects due to the torque generated by the torque motor. This force is the result of the current through the two coils fixed around the armature, which is connected to the flapper. As a 
result of this displacement, the cross sections of the orifices associated with the nozzles change. Schematic of SV cross section is shown in Fig. 3.

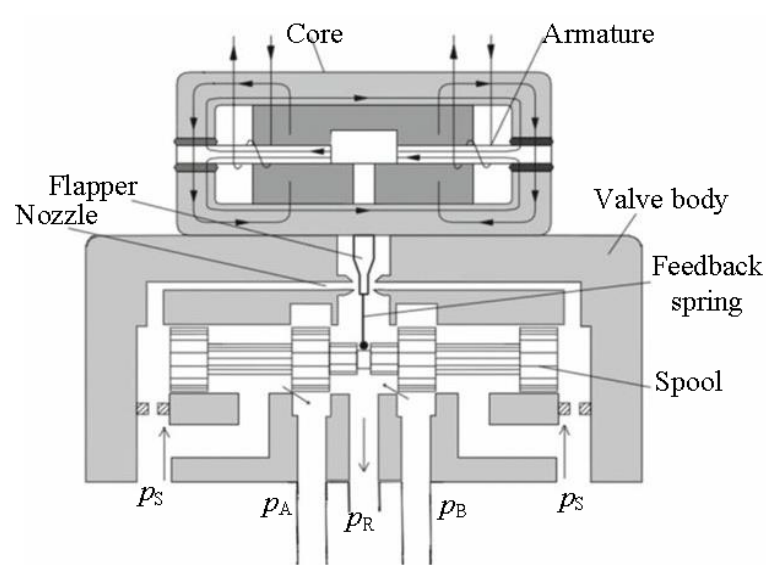

Fig. 3. Schematic of SV;

$\left(p_{\mathrm{S}}\right.$ is supply pressure, $p_{\mathrm{R}}$ return pressure,

$p_{\mathrm{A}}$ pressure in chamber $A$, and $p_{\mathrm{B}}$-pressure in chamber $\mathrm{B}$ )

Dynamic behaviour of valves involves a large number of parameters. An accurate analytical description would be time consuming and extremely difficult to validate for details. Therefore it is useful to use manufacturer's catalogue information which provides the well-known step responses and frequency responses for various sizes and types of valves. The inspection of step responses and frequency diagrams suggests an approximation of the SV by a second order model of the form [17].

$$
\frac{1}{\omega_{v}^{2}} \ddot{x}_{v}+\frac{2 \xi_{v}}{\omega_{v}} \dot{x}_{v}+x_{v}+f_{H S} \operatorname{sgn}\left(\dot{x}_{v}\right)=k_{v} \cdot u
$$

where $\omega_{v}$ is a valve natural frequency, $\xi_{v}$ damping coefficient, $x_{v}$ valve spool displacement, $f_{H S}$ takes into account the valve hysteresis and response sensitivity, $k_{v}$ valve flow gain, and $u$ valve input signal.

\subsection{Hydraulic Actuator Dynamics}

Hydraulic actuator (cylinder) converts hydraulic power into mechanical power. Hydraulic cylinder consists mainly of hollow cylindrical body and a piston. The chamber pressure dynamics is a wellknown expression proposed by [5] and can be obtained by applying the conversation principle to each individual chamber, as follows:

$$
\dot{p}_{A}=\frac{\beta_{\text {Aeff }}}{V_{A}}\left[Q_{A}-A_{p} \dot{x}_{p}+Q_{L i}-Q_{L e A}\right],
$$

$$
\dot{p}_{B}=\frac{\beta_{B e f f}}{V_{B}}\left[Q_{B}+\alpha A_{p} \dot{x}_{p}-Q_{L i}-Q_{L e B}\right]
$$

The effective bulk modulus essentially depends on pressure, entrained air and mechanical compliance. The commonly used empirical equation for calculation of the effective bulk modulus for hydraulic cylinders is expressed as [17]:

$$
\begin{aligned}
& \beta_{\text {Aeff }}=a_{1} \beta_{\max } \cdot \log \left(a_{2} \frac{p_{A}}{p_{\max }}+a_{3}\right), \\
& \beta_{\text {Beff }}=a_{1} \beta_{\max } \cdot \log \left(a_{2} \frac{p_{B}}{p_{\max }}+a_{3}\right),
\end{aligned}
$$

with the parameters $a_{1}=0.5, \quad a_{2}=90, a_{3}=3$, $\beta_{\max }=18000$ [bar], $p_{\max }=280$ [bar].

The cylinder chambers volumes are given by:

$$
\begin{gathered}
V_{A}=V_{A 0}+\left[A_{p}\left(x_{0}+x_{p}\right)\right], \\
V_{B}=V_{B 0}+\left[\alpha A_{p}\left(x_{0}-x_{p}\right)\right],
\end{gathered}
$$

where $x_{0}$ is initial piston position, $x_{p}$ actual piston position and $V_{A 0}, V_{B 0}$ are the initial chamber volumes which consist of an efficient part (the volume required to fill only the chambers) and inefficient part (volume of the pipelines between the valve and actuator) for the side $A$ and $B$ respectively. $A_{A}=A_{p}$ is a piston surface area and the $A_{B}=\alpha A_{p}$ rode side area where $\alpha=A_{B} / A_{A}$ is a ratio of cylinder bore area and the annulus effective area at the rod side.

$Q_{L i}$ and $Q_{L e}$ denote the internal leakage flow and the external leakage flow respectively. Leakage from one cylinder chamber to another, known as internal leakage flow, by assumption of laminal flow can be calculated:

$$
Q_{L i}=k_{L}\left(p_{B}-p_{A}\right),
$$

where $k_{L}$ is internal leakage flow coefficient, while the external leakage can be neglected.

The mechanical subsystem dynamics of the piston and the moving mass is described by dynamic equations:

$$
m_{t} \cdot \ddot{x}_{p}+F_{f}\left(\dot{x}_{p}\right)=\left(p_{A}-\alpha \cdot p_{B}\right) A_{p}-F_{e x t},
$$

where $m_{t}$ is total mass, $F_{f}$ friction force and $F_{e x t}$ external load force.

The total mass $m_{t}$ consists of the piston mass and the mass of hydraulic fluid in the cylinder chambers 
and the pipelines. However, mass of fluid can usually be neglected with the piston mass.

An important part of above equation is a friction force. Friction is a complex natural phenomenon. It occurs at the physical interface of two surfaces in contact and tangential reaction between them. It can lead to tracking errors, limit cycle oscillations and undesirable stick-slip motion. Friction is commonly modelled as a discontinuous static mapping between the velocity and the friction force:

$$
\begin{gathered}
F_{f}\left(\dot{x}_{p}\right)=F_{v}\left(\dot{x}_{p}\right)+F_{c}\left(\dot{x}_{p}\right)+F_{s}\left(\dot{x}_{p}\right)= \\
=\sigma_{v f} \cdot \dot{x}_{p}+F_{c o} \cdot \operatorname{sgn}\left(\dot{x}_{p}\right)+\operatorname{sgn}\left(\dot{x}_{p}\right) \cdot F_{s o} \cdot e^{-\frac{\left|\dot{x}_{p}\right|}{c_{s}}},
\end{gathered}
$$

with $\sigma_{v f}$ is the parameter for viscus friction, $F_{c o}$ static friction force, $F_{s o}$ Stribeck friction force, $c_{s}$ Stribeck velocity. This friction force depends on the velocity sign and is restricted to viscous friction and Coloumb friction with Stribeck effect. Moreover, the friction force must be considered in order to obtain acceptable tracking accuracy.

Natural frequency can be also calculated based on the system design parameters. The natural frequency for the overall system equals the natural frequency of the hydro-mechanical part. Therefore, an electrohydraulic control system natural frequency will be:

$$
\omega_{H}=\sqrt{\frac{C_{H}}{m}} .
$$

In order to calculate the natural frequency of hydraulic cylinder, the hydraulic cylinder stiffness must be found in advance. The total stiffness of differential cylinder is defined with equation [16]:

$$
C_{H}=\frac{\beta_{\text {Aeff }} \cdot A_{A}^{2}}{V_{A}}+\frac{\beta_{\text {Beff }} \cdot A_{B}^{2}}{V_{B}} .
$$

In case of synchronous cylinder (double rod of equal area) $A_{A}$ is equal $A_{B}$. For performance calculation, only the minimum stiffness will be considered since it has the worst effect on the system dynamics.

\subsection{Model Simplification}

For the purpose of design of a closed loop controller the complete mathematical model of the system which consists of the hydraulic actuator dynamics, including the load and SV dynamics can be simplified according to manufacturers data of real system components. Usually a simplification can be obtain when the dynamic of the servo valve with control electronics is much higher than the dynamic of the cylinder and load.

For hydraulic system with $A_{p}=6.4 \cdot 10^{-4} \mathrm{~m}^{2}$, $V_{t}=131.85 \cdot 10^{-6} \mathrm{~m}^{3}$ and $m=200 \mathrm{~kg}$ the minimum natural frequency is:

$\omega_{H \min }=\sqrt{\frac{2 \beta \cdot A_{p}^{2}}{m \cdot V_{t}}}=215.866\left[\frac{\mathrm{rad}}{\mathrm{s}}\right] \Rightarrow 34.35[\mathrm{~Hz}]$.

Natural frequency can be read out, from the manufacturers data sheet for SV MOOG 769 [18] we can read out $f_{H S v}=325 \mathrm{~Hz}$.

Because of very high natural frequency of servo valve with comparison to hydraulic cylinder $\left(\omega_{H s v}>>\omega_{H m i n}\right)$, taking in to account also dynamic effect of manifold which decreases hydraulic natural frequency, the dynamic of SV can be neglected. The servo valve can be described only by static relationship between spool position and the valve current input. Moreover the combined assembly of servovalve and electronic amplifier can be described by the equation:

$$
x_{v}=k_{v} \cdot u \text {, }
$$

where $k_{v}\left[\mathrm{mV}^{-1}\right]$ is combined $\mathrm{SV}$ and electronic amplifier gain. Therefore the simplified system equations have the following form:

$\dot{x}_{p}=v_{p}$,

$\dot{v}_{p}=\frac{A_{p}}{m} p_{L}-\frac{F_{e x t}}{m}-$

$-\frac{1}{m}\left[\sigma_{v f} \cdot \dot{x}_{p}+F_{c o} \cdot \operatorname{sgn}\left(\dot{x}_{p}\right)+\operatorname{sgn}\left(\dot{x}_{p}\right) \cdot F_{s o} \cdot e^{-\frac{\left|\dot{x}_{p}\right|}{c_{s}}}\right]$,

$\dot{p}_{L}=\frac{4 \beta}{V_{t}} \cdot C_{d} \cdot k_{v} \sqrt{\frac{p_{s}-\operatorname{sgn}(u) p_{L}}{\rho}} \cdot u-\frac{4 \beta}{V_{t}}$.

$\cdot A_{p} \cdot \dot{x}_{p}-\frac{4 \beta}{V_{t}} \cdot k_{L} \cdot p_{L}$.

where $x_{p}$ is actual piston position, $v_{p}$ piston velocity and $p_{L}$ load pressure.

\section{CONTROLLER DESIGN}

For a spatial class of nonlinear dynamical systems, the backstepping control has emerged us successful control strategy. Some of nonlinear systems can be observed as a systems constructed from subsystems. The number of subsystems depends of dynamic model order. Because of this recursive structure, the designer can start the design process at the knownstable system and design,"back out", new controllers 
that progressively stabilize each outer subsystem. The process is finished when the final external control is reached.

The mathematical description of nonlinear system in new set of coordinates allows us to use the backstepping approach to force it to behave like a linear one [7]. Backstepping has the availability to avoid cancellations of useful nonlinearities and pursue the objectives of stabilization and tracking rather than that of linearization method. Besides, backstepping approaches relaxes the matching conditions on perturbations which means that the perturbation doesn't have to appear in the equation that contains the input of the system. This controller can be used for tracking and regulation problem. For tracking problem, backstepping always use the error between the actual and desired input in order to start the design process.

The system state equations can be represented in "strict feedback form":

$$
\begin{aligned}
& \dot{x}_{1}=x_{2}, \\
& \dot{x}_{2}=a_{1} x_{3}-a_{2} x_{2}-a_{3} \operatorname{sgn}\left(x_{2}\right)-a_{4}, \\
& \dot{x}_{3}=a_{5} \sqrt{p_{s}-\operatorname{sgn}(u) x_{3}} u-a_{6} x_{2}-a_{7} x_{3},
\end{aligned}
$$

where $x_{1}=x_{\mathrm{p}}, x_{2}=v_{\mathrm{p}}$ and $x_{3}=p_{\mathrm{L}}$, with:

$$
\begin{aligned}
& a_{1}=\frac{A_{p}}{m}, \quad a_{2}=\frac{\sigma_{v f}}{m}, \quad a_{3}=\frac{F_{c o}}{m}, \quad a_{4}=\frac{F_{e x t}}{m}, \\
& a_{5}=\frac{4 \beta}{V_{t} \sqrt{\rho}} \cdot C_{d} \cdot k_{v}, \quad a_{6}=\frac{4 \beta}{V_{t}} \cdot A_{p} \quad a_{7}=\frac{4 \beta}{V_{t}} \cdot k_{L} .
\end{aligned}
$$

In the procedure of design a backstepping controller it is preferable to replace nonlinear terms with differentiable mathematical functions. An essential factor of its validity is the continuous dependence of its solutions on the data of the problem that can be stated by the Lipschitz inequality given by:

$$
\|f(x)-f(y)\| \leq L\|x-y\|
$$

To satisfy condition in Eq. (17) we estimated the "sgn" function with continuously differentiable function "tanh $=t h$ " (hyperbolic tangent) except in last equation.

$$
\begin{gathered}
\dot{x}_{1}=x_{2}, \\
\dot{x}_{2}=a_{1} x_{3}-a_{2} x_{2}-a_{3} \operatorname{th}\left(\lambda x_{2}\right)-a_{4}, \\
\dot{x}_{3}=a_{5} u \sqrt{p_{s}-\operatorname{sgn}(u) x_{3}}-a_{6} x_{2}-a_{7} x_{3}
\end{gathered}
$$

where $\lambda$ is free coefficient $(\lambda=2)$
The control objectives were to stabilize the plant and to track the given reference signal asymptotically. The detailed derivations of finding backstepping control input are covered in next three steps [9].

\section{Step 1}

We want the $x_{1}$ is position variable to track a reference signal, say, $r(t)$. So we have the first error variable:

$$
z_{1}=x_{1}-r
$$

and its derivative:

$$
\dot{z}_{1}=\dot{x}_{1}-\dot{r}
$$

where $r(t)$ is the reference input.

For equation (18a) we can define a virtual control $\alpha_{1}$. Virtual state variable $z_{2}$ represents the difference between the actual and virtual control of (18a), i.e.

$$
z_{2}=x_{2}-\alpha_{1} \rightarrow x_{2}=z_{2}+\alpha_{1} .
$$

Define a candidate control Lyapunov functional for this equation:

$$
V_{1}=\frac{\rho_{1}}{2} \cdot z_{1}^{2}
$$

The derivative of $V$ yields:

$$
\dot{V}_{1}=\rho_{1} \cdot\left[\left(x_{1}-r\right) \cdot z_{2}+\left(x_{1}-r\right)\left(\alpha_{1}-\dot{r}\right)\right] \text {. }
$$

Select a virtual control for the first order system:

$$
\alpha_{1}=\dot{r}-K_{1} z_{1}=-K_{1} x_{1}+K_{1} r+\dot{r},
$$

where $K_{1}>0$. Hence, $\dot{V}$ can be rewritten as:

$$
\dot{V}_{1}=-\rho_{1} K_{1} z_{1}^{2}+\rho_{1} z_{1} z_{2} .
$$

\section{Step 2}

Define a second virtual state

$$
z_{3}=x_{3}-\alpha_{2} \quad \rightarrow \quad x_{3}=z_{3}+\alpha_{2},
$$

thus:

$$
\dot{z}_{2}=\dot{x}_{2}-\dot{\alpha}_{1}
$$

Define a candidate control Lyapunov functional for this equation:

$$
V_{2}=V_{1}+\frac{\rho_{2}}{2} \cdot z_{2}^{2}
$$

The derivative of $V_{2}$ is:

$$
\dot{V}_{2}=\dot{V}_{1}+\rho_{2} \cdot z_{2} \cdot \dot{z}_{2}
$$


Select a virtual control for this system to remove any potentially undesired $z_{2}, x_{1}$ and $x_{2}$ terms:

$$
\alpha_{2}=\frac{1}{a_{1}}\left(a_{2} x_{2}+a_{3} t h\left(\lambda x_{2}\right)+a_{4}+\dot{\alpha}_{1}-\frac{\rho_{1}}{\rho_{2}} z_{1}-\frac{K_{2}}{\rho_{2}} z_{2}\right),
$$

where $K_{2}>0$, therefore:

$$
\dot{V}_{2}=-K_{1} \cdot \rho_{1} \cdot z_{1}^{2}-K_{2} \cdot z_{2}^{2}+\rho_{2} \cdot a_{1} \cdot z_{2} \cdot z_{3},
$$

\section{Step 3}

Let's recall $z_{3}=x_{3}-\alpha_{2}$. The derivative of $z_{3}$ is:

$$
\dot{z}_{3}=\dot{x}_{3}-\dot{\alpha}_{2}
$$

With augmentation of the initial control Lyapunov functional to reflect the presence of the new state variable:

$$
V_{3}=V_{2}+\frac{\rho_{3}}{2} \cdot z_{3} .
$$

The derivative yields:

$$
\dot{V}_{3}=\dot{V}_{2}+\rho_{3} \cdot z_{3} \cdot \dot{z}_{3} .
$$

Select an actual control for this system:

$$
\begin{aligned}
& u=\frac{1}{\rho_{3} a_{5}} \frac{1}{\sqrt{p_{s}-x_{3}}} \cdot \\
& \cdot\left[-\rho_{2} a_{1} z_{2}+\rho_{3} a_{6} x_{2}+\rho_{3} a_{7} x_{3}+\rho_{3} \dot{\alpha}_{2}-K_{3} z_{3}\right],
\end{aligned}
$$

where $K_{3}>0$. Then we get:

$$
\dot{V}_{3}=-K_{1} \cdot \rho_{1} \cdot z_{1}^{2}-K_{2} \cdot z_{2}^{2}-K_{3} z_{3}^{2},
$$

where $K_{1}, K_{2}, K_{3}>0$. Note that Eq.(28) is the Lyapunov function of the system defined by Eqs.(18a) to (18c)

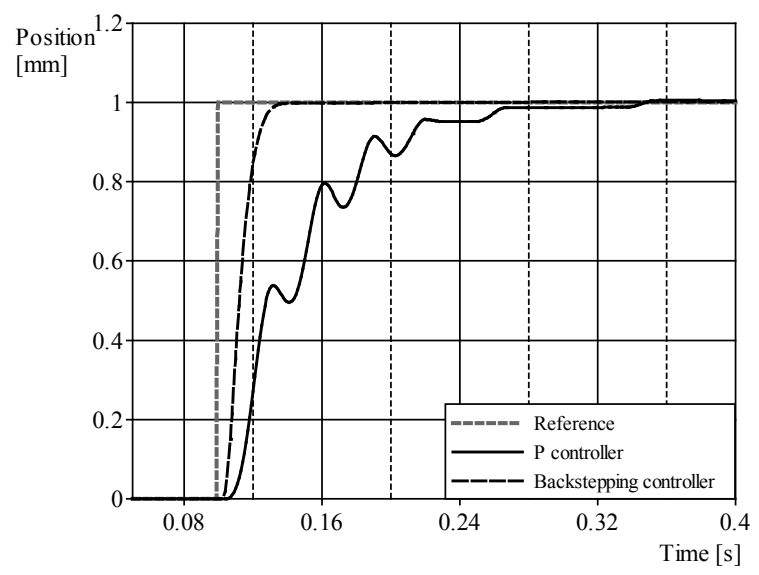

Fig. 4. Simulation results of the system step response and that the control law given by Eqs. (24), (30) and (35) renders its derivative negative semidefinite.

\section{RESULTS AND DISCUSIONS}

In order to verify the effectiveness of the proposed backstepping control algorithm, simulation experiments have been done on the electrohydraulic servo system. Main parameters of the system are given in Table1.

For position control systems dynamically aperiodic response is demanded. The final position accuracy is tested at small steps of reference value.

In Fig. 4 the comparison of systems dynamic response between P-controller and backstepping

\begin{tabular}{|c|c|c|c|}
\hline Par. & Value & Par. & Value \\
\hline$A_{p}$ & $6.4 \cdot 10^{-4} \mathrm{~m}^{2}$ & $m$ & $200 \mathrm{~kg}$ \\
\hline$\sigma_{v f}$ & $70 \mathrm{Ns} / \mathrm{m}$ & $V_{t}$ & $131.85 \cdot 10^{-6} \mathrm{~m}^{3}$ \\
\hline$F_{c o}$ & $19.62 \mathrm{~N}$ & $\beta$ & $1.5 \cdot 10^{9} \mathrm{~Pa}$ \\
\hline$F_{\text {ext }}$ & {$[\mathrm{N}]$} & $p_{s}$ & 210 bar \\
\hline$k_{L}$ & $3 \cdot 10^{-13} \mathrm{~m} 5 / \mathrm{Ns}$ & $\rho$ & $850 \mathrm{~kg} / \mathrm{m}^{3}$ \\
\hline$C_{d}$ & 0.63 & $k_{V}$ & $5.53 \cdot 10^{-7} \mathrm{~m}^{2} / \mathrm{V}$ \\
\hline
\end{tabular}
controller to $1 \mathrm{~mm}$ desired position step is shown. The corresponding tracking errors are shown on Fig. 5. The system state variables are shown on Fig. 6, while corresponding virtual control variables and final real control of the backstepping controller are shown on Fig. 7.

Table 1. Main parameters of the EHS system

In Fig. 8 the comparison of systems dynamic response to sinusoidal reference input is shown. The corresponding tracking errors are shown on Fig. 9. The system state variables are shown on Fig. 10,

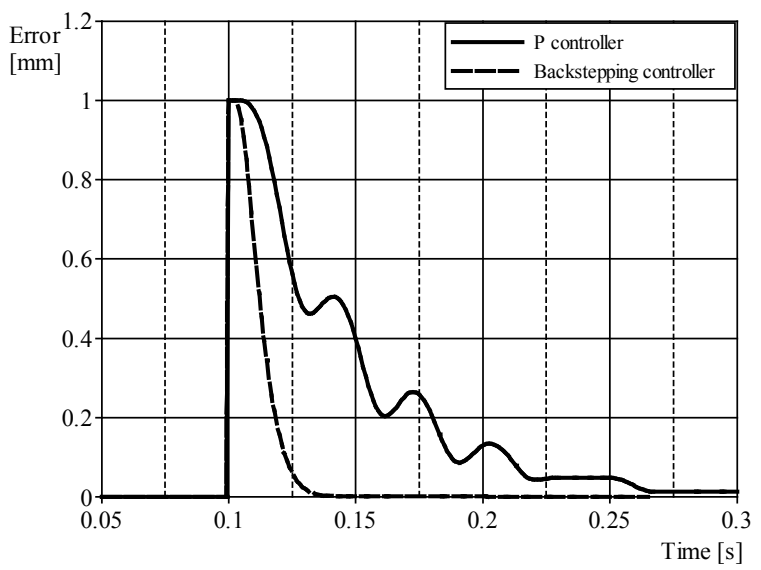

Fig. 5. Tracking error of the system step response 


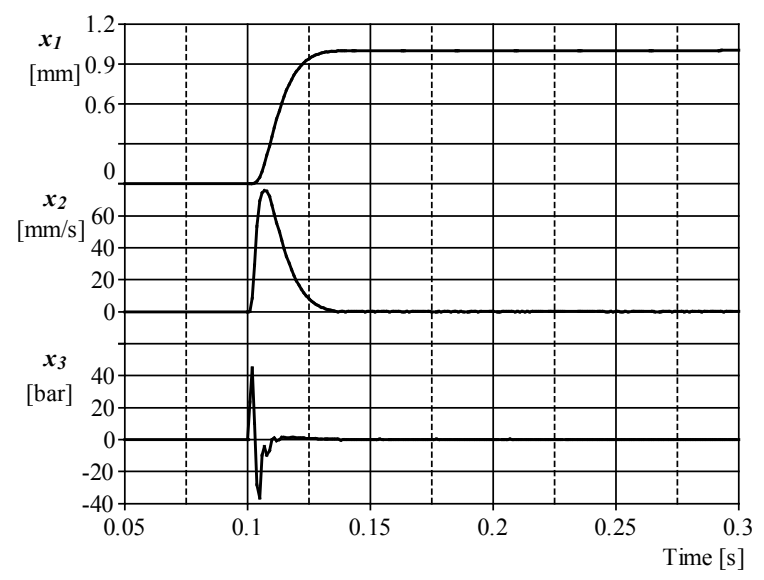

Fig. 6. Dynamic behaviour of the systems variables by step response

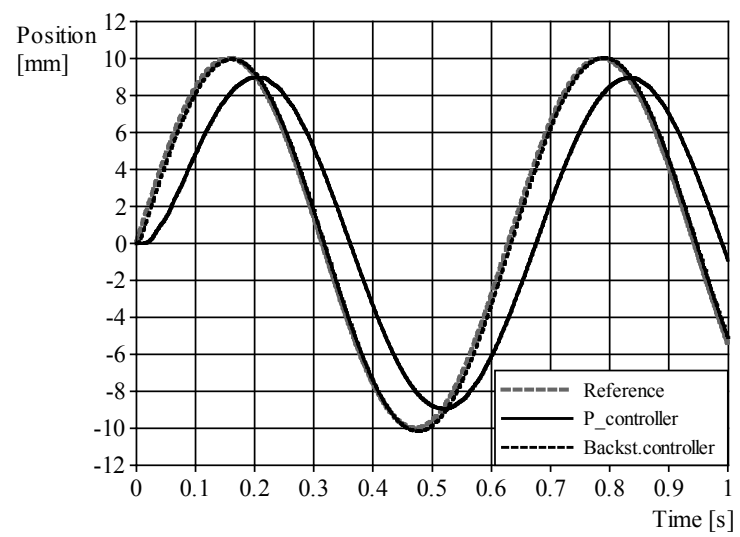

Fig. 8. Simulation results of the system response on sinusoidal reference input

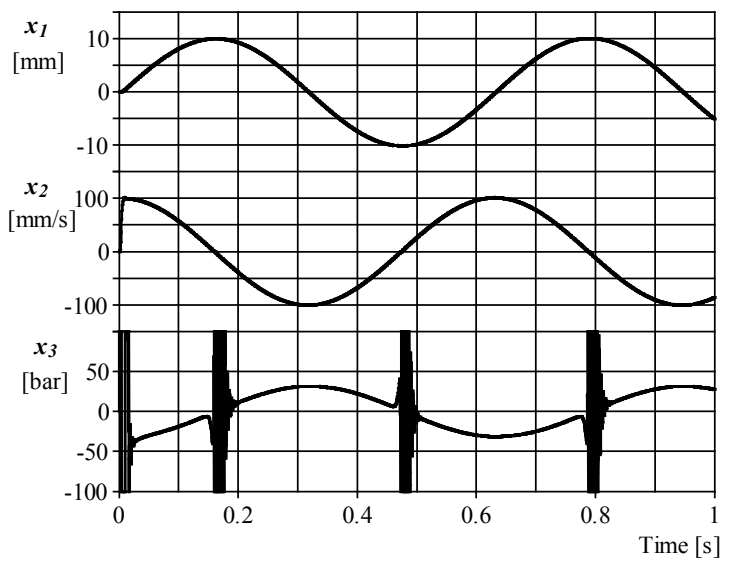

Fig. 10. Dynamic behaviour of the systems variables at sinusoidal reference input

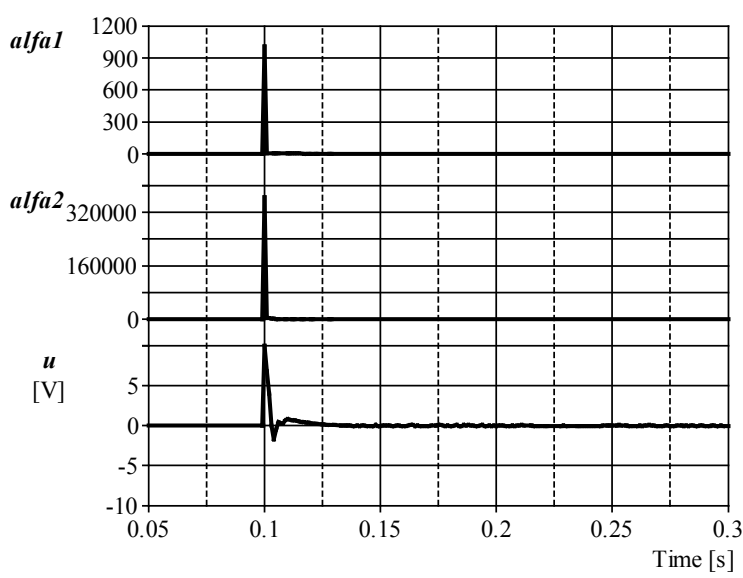

Fig. 7. Virtual control variables and final real control variable of backstepping controller

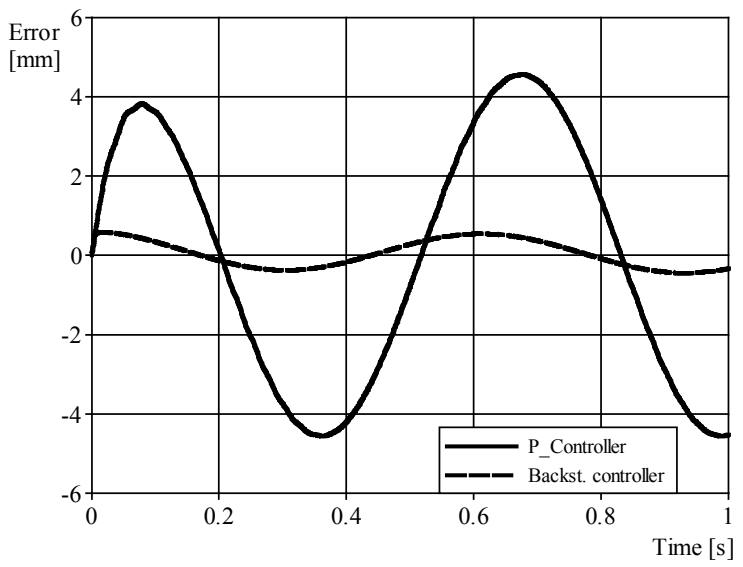

Fig. 9. Tracking error of the system response on sinusoidal reference input

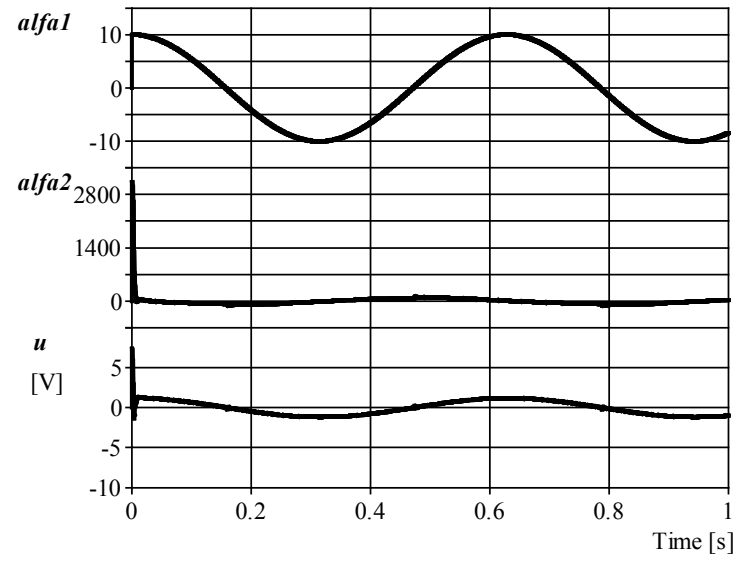

Fig. 11. Virtual control variables and final real control variable of backstepping controller 
while corresponding virtual control variables and final real control of the backstepping controller are shown on Fig. 11.

The results are obtained with P-controller gain $K_{P}=2.3$, while the backstepping controller parameters are $K_{1}=50, K_{2}=600, K_{3}=600, \rho_{1}=6500, \rho_{2}=0.65$, $\rho_{3}=1$.

\section{CONCLUSIONS}

In this paper, a controller design procedure is presented in order to control the position of the electrohydraulic servo system. Comprehensive investigation was carried out on the mathematical modelling and computer simulation of dynamic behaviour of whole system, based on real laboratory experimental data.

Because of very fast SV in combination with large moving mass and relatively long hydraulic cylinder, the mathematical model was reduced to third order, neglecting SV first stage dynamics. Such simplified mathematical model was used as a basis to design the closed loop controller. The developed control strategy is based on Lyapunov stability theory of nonlinear systems. We proposed a robust nonlinear controller, using integrator backstepping approach. The validity of the proposed new feedback law has been tested and compared with P-controller in various computer simulations. In all cases the nonlinear controller shows best tracking performance, with very small position tracking error. Excellent tracking capability of the proposed new controller is also recognized by sinusoidal reference input. Furthermore the tracking errors converge to zero quickly in contrast to the non-zero tracking error of the P-controller. The new controller also shows a certain measure of robustness to small parameter changes. Finally, the controller guaranties a prescribed transient performance and final tracking accuracy. Our future work will look towards the development of nonlinear adaptive controller which would be able to work in the presence of parametric uncertainties and environmental changes.

\section{REFERENCES}

[1] Khalil, H.K. (2009). Interactive analysis of closed loop electrohydraulic control systems, 13th International Conference on Aerospace Scienties \& Aviation Technology, ASAT-13-HC-01.

[2] Kovari, A. (2015). Effect of Leakage i Electrohydraulic Servo Systems Based on Complex Nonlinear Mathematical Model and Experimental Results. Acta Polytechnica Hungarica, vol. 12, no. 3, p. 129-146, DOI:10.12700/APH.12.3.2015.3.8.

[3] Gubeljak, N., Predan, J., Senčič, B., Chapetti, M.D. (2014). Effect of residual stresses and inclusion size on fatigue resistance of parabolic steel springs. Materials Testing, vol. 56, no. 4, p. 312-317, Dol:10.3139/120.110567.

[4] Veg, E., Veg, A., Sinkovic, G., Andrejevic, R., Gubeljak, N. (2015). Design of coupled slider crank mechanism for orbiting motion, International Journal of Simulation Modelling, vol. 14, no. 2, p. 189-200, DOI:10.2507/IJSIMM14(2)1.283.

[5] Merritt, H.E. (1967). Hydraulic Control Systems. Wiley, NewYork.

[6] Lovrec, D., Tic, V., Tasner, T. (2015). Simulation-aided determination of an efficiency field as a basis for maximum efficiency controller design. International Journal of Simulation Modelling, vol. 14, no. 4, p. 669-682, Dol:10.2507/ IJSIMM10(3)3.184.

[7] Kristic, M., Kanellakopouls, I., Kokotovic, P.V. (1995). Nonlinear and Adaptive Control Design, John Wiley and Sons, Hoboken.

[8] Khalil, H. K. (2002). Nonlinear Systems, 3 $3^{\text {rd }}$ ed., Prentice Hall, Upper Saddle River.

[9] Lee, S. J., Tsao, T.-C. (2002). Nonlinear backstepping control of an electrohydraulic material testing system, Proceedings of the American Control Conference, vol 6. p. 4852-4830, DOI:10.1109/ACC.2002.1025422.

[10] Ursu, I., Ursu, F., Popescu, F. (2006). Backstepping design for controlling electrohydraulic servos. Journal of the Franklin Institute, vol. 343, no. 1, p. 94-110, D0l:10.1016/j. jfranklin.2005.09.003.

[11] Bonchis, A., Corke, P.I., Rye, D.C., Ha, Q.P. (2001) Variable structure methods in hydraulic servo systems control. Automatica, vol.37, no. 4, p. 589-95, D0l:10.1016/S00051098(00)00192-8.

[12] Nakkarat, P., Kuntanapreeda, S. (2009). Observer-based backstepping force control of an electrohydraulic actuator. Control Engineering Practice, vol. 17, no. 8, p. 895-902, D0l:10.1016/j.conengprac.2009.02.011.

[13] Sun,W., Gao, H., Kaynak, O. (2013). Adaptive Backstepping Control for Active Suspension Systems With Hard Constraints, IEEE/ASME Transactions on Mechatronics, vol. 18, no. 3, p. 1072-1079, DOI:10.1109/TMECH.2012.2204765.

[14] Wonohadidjojo, D.M., Kothapalli, G., Hassan, M.Y. (2013). Position Control of Electro-hydraulic Actuator System Using Fuzzy Logic Controller Optimized by Particle Swarm Optimization. International Journal of Automation and Computing, vol. 10, no. 3, p.181-193, Dol:10.1007/s11633013-0711-3.

[15] Lovrec, D., Kastrevc, M. (2011). Modelling and simulating a controlled press-brake suplay system. International Journal of Simulation Modelling, vol. 10, no. 3, p. 133-144, D0l:10.2507/ IJSIMM10(3)3.184.

[16] Wright, H., Alleyne, A., Liu, R. (1997). On the stability and performance of two-state hydraulic servovalves. Proceedings of the ASME Dynamic Systems and Control Division, vol. 63, p. 215-222.

[17] Jelali, M., Kroll, A. (2003). Hydraulic Servo-systems:modelling, identification and control. Springer-Verlag, London, Berlin, Heidelberg, Dol:10.1007/978-1-4471-0099-7.

[18] MOOG. Servovalves with Integrated Electronics D769 Series. Rapport technique, MOOG Inc., from http:/www.moog.com/ literature/ICD/D769seriesvalves.pdf, accessed on 2015-0603. 